Research Article

\title{
Research on the Three-Dimensional Power Frequency Electric Field Measurement System
}

\author{
Chunguang Suo, ${ }^{1}$ Ran Wei, ${ }^{1}$ Wenbin Zhang $\mathbb{D}^{\circ},{ }^{2}$ and Yincheng $\mathrm{Li} \mathbb{D}^{1}$ \\ ${ }^{1}$ Faculty of Science, Kunming University of Science and Technology, Kunming 650504, China \\ ${ }^{2}$ Faculty of Mechanical and Electrical Engineering, Kunming University of Science and Technology, Kunming 650504, China
}

Correspondence should be addressed to Wenbin Zhang; zwbscg@kust.edu.cn and Yincheng Li; liyincheng@stu.kust.edu.cn

Received 29 September 2020; Revised 29 August 2021; Accepted 2 September 2021; Published 20 September 2021

Academic Editor: Rosalba Liguori

\begin{abstract}
Copyright (c) 2021 Chunguang Suo et al. This is an open access article distributed under the Creative Commons Attribution License, which permits unrestricted use, distribution, and reproduction in any medium, provided the original work is properly cited.
\end{abstract}

\begin{abstract}
It is of great significance to accurately provide electric field information for electric power operators and electric power inspection equipment to ensure the safety of live working robots and electric power workers. In this paper, the principle of parallel plate charge induction is used to detect electric field signals and the influence of the inherent capacitance of the parallel plate on the back-end circuit is considered. An equipotential ring is used as the structure of the sensing unit to eliminate the uncertainty generated by the edge electric field. The parameters of the sensor probe are determined by the principle of differentialintegration, and its structure is analyzed and designed. The consistency of the designed sensor probe was analyzed, and the results show that the consistency of the sensor is better. We have measured the sensitivity coefficients of the six probes, and the average absolute deviation reached 0.031556. The fits were all above 0.9995. In addition, a three-dimensional power frequency electric field measurement system that is easy to manufacture is designed using the hexahedral structure, which solves the problem of inaccurate electric field measurement caused by the parallel plate probe and the field source being not perpendicular, and the combined field strength formula of nonuniform electric field was obtained. In the laboratory environment, the three-dimensional power frequency electric field measurement system produced in this paper is compared with the electric field simulated by an electric field simulation tool. The test results show that the deviation between the measurement system and simulation is within $\pm 0.55 \%$, the measurement range is $1 \mathrm{kV} / \mathrm{m}-200 \mathrm{kV} / \mathrm{m}$, the resolution is $\geq 1 \mathrm{~V} / \mathrm{m}$, and the maximum electric field can be measured at $200 \mathrm{kV} / \mathrm{m}$. The nonlinear error is $2.15 \%$, and the sensitivity coefficient is $19.10 \mathrm{mV} /\left(\mathrm{kV} \cdot \mathrm{m}^{-1}\right)$, which meets the measurement requirements of the power frequency electric field and can be applied to the actual power frequency electric field measurement.
\end{abstract}

\section{Introduction}

Electric field measurement is of great significance in many fields of scientific research and engineering technology, such as the measurement of the pulsed electric field of defense technology nuclear explosion, the real-time monitoring of lightning transient electric field in the aerospace field, the field detection of medical equipment in the power medical industry, and the field of communication near-field measurement of mid-antenna and biological effects of the bioelectric field [1-5]. The electric field measurement technology gives strong support for the above fields, especially for the power systems [6-8].
There are many companies or teams in the world that have conducted in-depth research on power frequency electric field sensors. For example, researchers in the United States were using the linear photoelectric effect of certain crystals (such as lithium niobate $\mathrm{LiNbO}_{3}$ and lithium tantalate $\mathrm{LiTaO}_{3}$ ) to linearly modulate the refractive index of the crystal by the external electric field, that is, the Pockels effect, which causes the optical signal in the product body to change in phase [9]. According to this principle, using proton exchange or titanium diffusion technology to form a stripe optical waveguide with a Mach-Zehnder (MZ) structure on the crystal surface, thereby converting the phase change into an easily measured optical power change, to 
achieve the measurement of the electric field [9-11], optical electric field sensors have wide bandwidth and fast response but the manufacturing process is complicated and costly. For some examples, reference [12] adopts an electric fieldsensitive microelectromechanical system (MEMS) to measure the induced current and realizes the measurement of the power frequency electric field. Reference [13] based on a silicon-on-insulator (SOI) technology MEMS resonant microsensitive structure measures the induced current and realizes the detection of the electric field. Reference [14] proposed a new atmospheric electric field sensor based on a high-sensitivity resonant MEMS electric field sensor chip, which solves the problems of motor wear, high power consumption, and failure rate. However, using MEMS to produce the electric field sensor is complicated and the manufacturing cost is relatively high. In reference [15], Feser and Pfaff of the University of Stuttgart in Germany, etc., improved the original spherical sensor and developed a two-dimensional spherical transient electric field measuring instrument using optical fiber as data transmission and isolating high voltage. In reference [16], the Swiss company Haefely also developed a two-dimensional spherical electric field instrument with a diameter of $8 \mathrm{~cm}$ and a measurement bandwidth of $20 \mathrm{MHz}$. In reference [17], researchers developed a high-precision two-dimensional spherical electric field measurement system. The system measures the electric field strength in the range of $0 \sim 100 \mathrm{kV} / \mathrm{m}$, and the measurement uncertainty is within $1 \%$. The system measured the electric field intensity of the current transformer distributed on the central axis and achieved very good experimental results. The University of Stuttgart and Haefely have studied a three-dimensional electric field measuring instrument based on a two-dimensional electric field instrument [18]. A large number of calculations and theoretical analyses have been carried out on the structure of the probe to improve the measurement accuracy, and the measurement uncertainty is less than $2.5 \%$. If the power frequency electric field sensor of the ball probe is used to measure the electric field, the manufacturing process of the probe is more demanding and the uncertainty of the probes produced at different levels is larger, which is unsuitable for mass production.

The structure of the existing electric field sensors, such as a spherical structure, MEMS [19] or cylindrical structure electric field sensor, their sensor unit design, and manufacturing are very complicated, and some of the sensor structure is very expensive to handle, which is not conducive to mass production. Besides, the sensor has a large volume and is difficult to carry. So these sensors cannot be applied to some newer application scenarios, like when electric power workers are performing live work, they need to accurately monitor the electric field information of their location, which is used to set threshold alarms to ensure personal safety. Another example is when drone inspection of the high-voltage transmission line is limited by the unmanned aerial vehicle (UAV) vision system, it is impossible to accurately judge the distance of the drone relative to the line. In this way, it is necessary to monitor the electric field information of the location to correct the relative position of the high-voltage transmission line, and when the inspection robot is performing live work, it also needs this information to get its location. After being processed by certain algorithms, it can achieve accurate positioning. Therefore, an electric field sensor system with a small size, which is light weight, and which has low price is developed and the sensor system can be mounted on different platforms, can provide specific electric field information for the corresponding equipment, and can provide electric field strength information for the corresponding equipment requirements, so as to ensure the safety of live working robots and power workers is of great significance.

In this paper, the principle of parallel plate charge induction $[20,21]$ and the principle of differential integration are used to design the structure of the sensor probe and the probe is used to produce a three-dimensional power frequency electric field sensor with a simple structure, convenient production, low price, high accuracy, and good consistency. The sensor can adapt to different platforms. In a laboratory environment, a measurement system is used to measure the electric field generated by the powerfrequency high-voltage field source. Through the comparison of simulation and measured data, the results show that the uncertainty of the measurement system does not exceed $\pm 0.55 \%$. It can meet the measurement requirements of the power frequency electric field [22] and can be applied to the measurement of the actual power frequency electric field.

The first part of this article introduces the background. The second part analyzes the measurement principle and designs the parameters of the differential integration circuit. The third part analyzes and designs the probe of the onedimensional electric field sensor and designs the subsequent conditioning circuit. In the fourth part, the one-dimensional electric field sensor is summarized as a three-dimensional electric field sensor and the one-dimensional to threedimensional field strength is introduced. In the last part, the field measurements are compared with Narda sensors, and finally, the full results are summarized.

\section{Measuring Principle and Signal Distortion Analysis}

2.1. Principle of Electric Field Induction Measurement. The charge in the conductor will move under the action of the electric field, thereby generating an internal electric field inside the conductor. The directions of the internal electric field and the surrounding electric field are opposite, and the strength and frequency of the internal electric field are the same as those of the external electric field [23, 24]. As shown in Figure 1(a), if the conductor is divided into two separate parts, such as two hemispheres of a spherical sensor or two plates of a parallel plate sensor, under the action of an external electric field $E$, the two polar plates will induce charge $Q$ [25-27]; assuming that the areal density of the induced charge is $\sigma(t)$ and the dielectric constant of the medium between the plates is $\varepsilon$, then, the induced charge of the plate has the following relationship with the measured external electric field strength: 


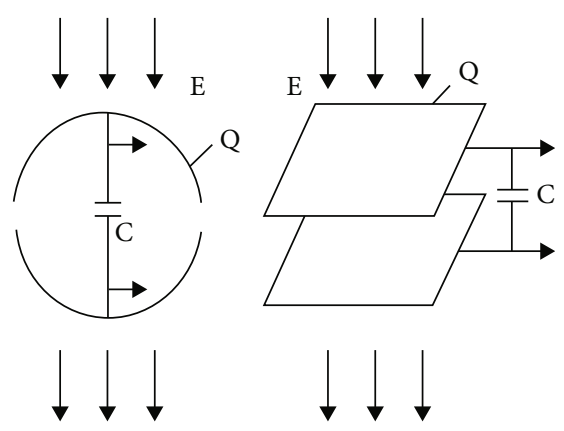

(a)

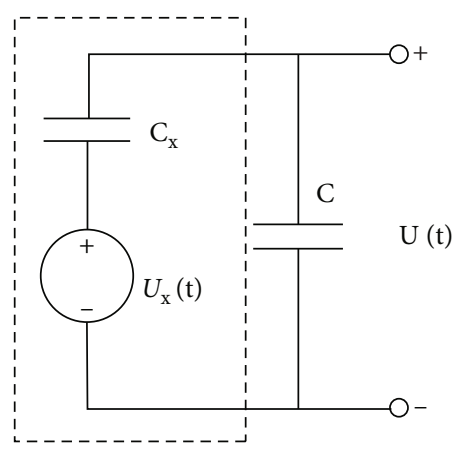

(b)

FIgURE 1: (a) Inductive electric field sensor probe and (b) equivalent circuit of its sensor measuring system.

$$
Q(t)=\int \sigma(\mathrm{t}) d S=\varepsilon E(t) S
$$

where $Q(t)$ is the amount of the induced charge generated by the electrode plate, $E(t)$ is the measured electric field strength, and $S$ is the effective area of the induced electrode plate.

The accumulation of the induced charge generated by the two plates creates a potential difference $U_{x}$ between the two plates. The change of the external electric field strength $E$ will cause the amount of induced charge $Q$ on the electrode plate to change. Since the internal charge cannot escape the restraint of the external electric field $E$, it can only move directionally inside the conductor, so it cannot form a current. By adding a sampling capacitor $C$ between the two plates, a voltage will form across the capacitor $C$. As long as the voltage across the capacitor $C$ is measured, the external electric field strength information can be derived.

Figure 1 shows the equivalent circuit of the induced electric field sensor probe and its sensor measurement system. In Figure 1(a), $E$ represents the external electric field, $Q$ is the surface-induced charge, and $C$ is the sampling capacitor; in Figure 1(b), $U_{x}(t)$ is the equivalent voltage source, $C_{x}$ is the inherent capacitance of the probe, $C$ is the sampling capacitance, and $U(t)$ is the output voltage.

The equivalent voltage source $U_{x}(t)$ is related to the sensor's inherent capacitance value and the amount of induced charge in the electric field:

$$
U_{x}(t)=\frac{Q(t)}{C_{x}}=\frac{\varepsilon E(t) S}{C_{x}}
$$

The upper and lower plates of the sensing unit are connected to the two ends of the sampling capacitor $C$. The voltage signal $U(t)$ formed on the sampling capacitor $C$ by the upper and lower sensing plates of the sensing unit is used as the output signal. The relationship is

$$
U(t)=\frac{k U_{x}(t)\left(C C_{x} / C+C_{x}\right)}{C}=\frac{k U_{x}(t) C_{x}}{C_{x}+C}=\frac{k Q(t)}{C_{x}+C},
$$

where $C_{x}$ is the inherent capacitance of the sensing unit, $k$ is the correction coefficient, and its value is related to the structure of the sensing unit. The calculation of the inherent capacitance of the parallel plate capacitor can be expressed as

$$
C_{\mathrm{x}}=\frac{\varepsilon \varepsilon_{0} S}{d}
$$

where $C_{x}$ is the inherent capacitance of the parallel plate capacitor, $d$ is the spacing between the plates, $\varepsilon$ is the dielectric constant of the medium between the plates, $\varepsilon_{0}$ vacuum dielectric constant is $8.86 \times 10^{-12} \mathrm{~F} / \mathrm{m}$, and $S$ is the induction plate effective area. Bring equations (1) and (4) into equation (3) for calculation and solution:

$$
U(t)=\frac{k \varepsilon \varepsilon_{r} E(t) S}{\left(\varepsilon \varepsilon_{r} S / d\right)+C}=\frac{k \varepsilon \varepsilon_{r} E(t) S d}{\varepsilon \varepsilon_{r} S+C d} .
$$

It can be known from equation (5) that as long as the voltage $U(t)$ across the sampling capacitor $C$ is measured, the electric field intensity $E$ at the measurement position can be obtained.

2.2. Distortion Analysis of the Power Frequency Electric Field Signal Measured by the Parallel Plate Probe. During the design of the power frequency electric field sensor probe, through a large number of actual measurements and tests, it was found that the measurement accuracy of the sensor using a parallel plate and box structure is very close to the measurement accuracy using a ball sensor. We use a $60 \mathrm{~Hz}$ power frequency high-voltage source for the test. For a parallel plate sensor, under a $18.3 \mathrm{kV} / \mathrm{m}$ uniform power frequency electric field, when the voltage across the two ends is directly measured with an oscilloscope, the waveform is distorted as shown in Figure 2. The peak-to-peak value of this waveform at $60 \mathrm{~Hz}$ is $113 \mathrm{mV}$.

To analyze the amplitude-frequency response, an FFT of the waveform is performed, as shown in Figure 3.

From Figure 3, it can be seen that the sensor signal contains the frequency component in $500 \mathrm{~Hz} 3500 \mathrm{~Hz}$. The fundamental frequency is highest at $60 \mathrm{~Hz}$, with the response reaching 0.1743 . These frequencies may be due to the measurement uncertainty of the probe or interference from other external factors. In order to get rid of them, we need to design a filter and the filter circuit will be designed below. 


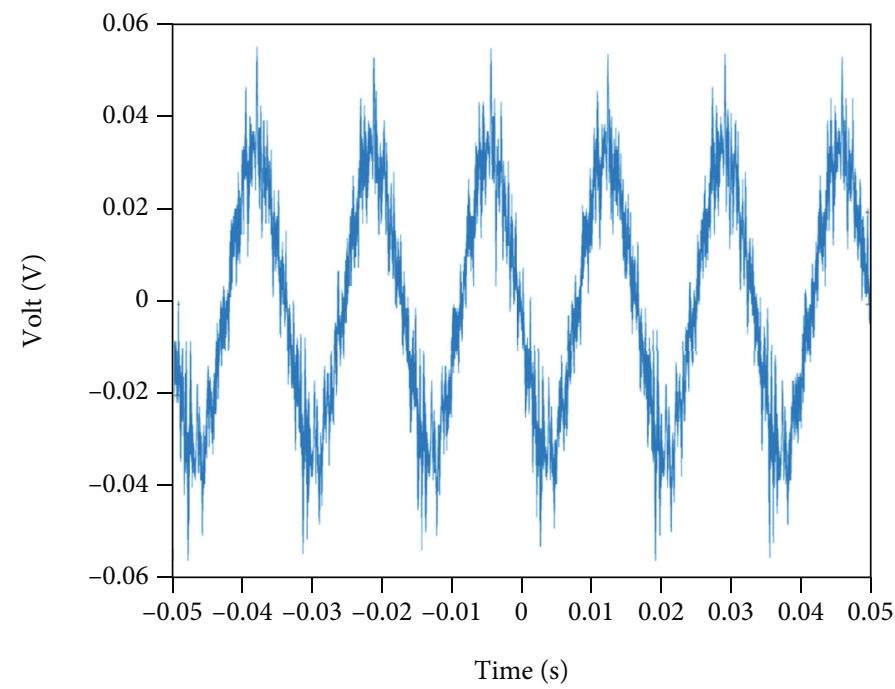

FIGURE 2: Waveform of voltage distortion.

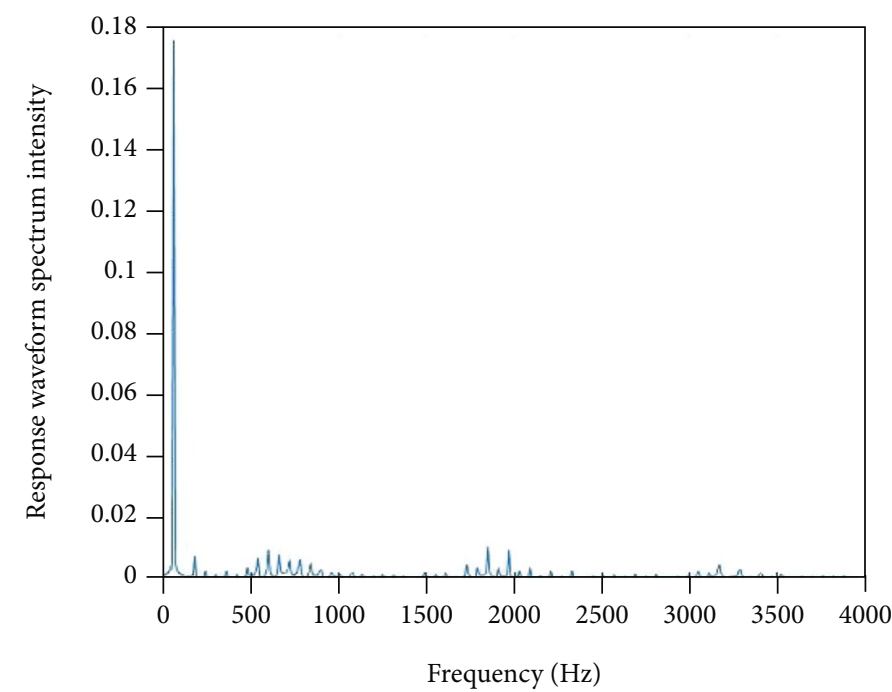

FIgURE 3: Amplitude frequency response characteristics analysis.

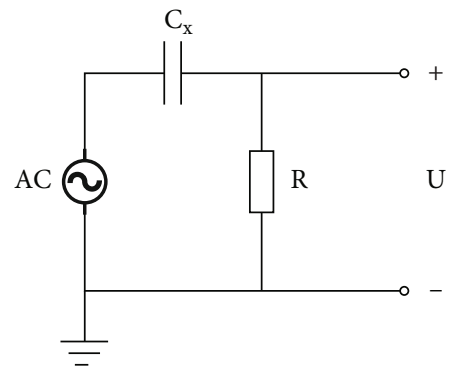

FIGURE 4: Equivalent circuit diagram.

For the effective filter design, we first need to analyze the equivalent circuit; considering the equivalent parallel internal resistance of the oscilloscope and the inherent capacitance in the sensor probe, the equivalent circuit is shown in Figure 4, where $C_{x}$ is the inherent capacitance in the sensor probe and $R$ is the internal equivalent parallel resistance of the oscilloscope. The inherent capacitance $C_{x}$ and the oscilloscope's internal equivalent parallel resistance $R$ form a differential circuit. Since the differential circuit outputs a differential signal, it needs to be recovered by an integrator and then output to a measuring device to measure the power frequency electric field. The internal resistance $R$ of the oscilloscope is $1 \mathrm{M}$ ohm.

\section{One-Dimensional Power Frequency Electric Field Measurement System}

3.1. Simulation Design of the Probe Structure of the Measurement System. According to the capacitance definition equation (4), the inherent capacitance of the sensor is related to the size, thickness, and internal filling material of the plate. And whether the measurement electrode of the parallel plate probe is parallel to the high-voltage field source affects the measurement of the electric field strength, the highest signal will be produced if the electric field is 


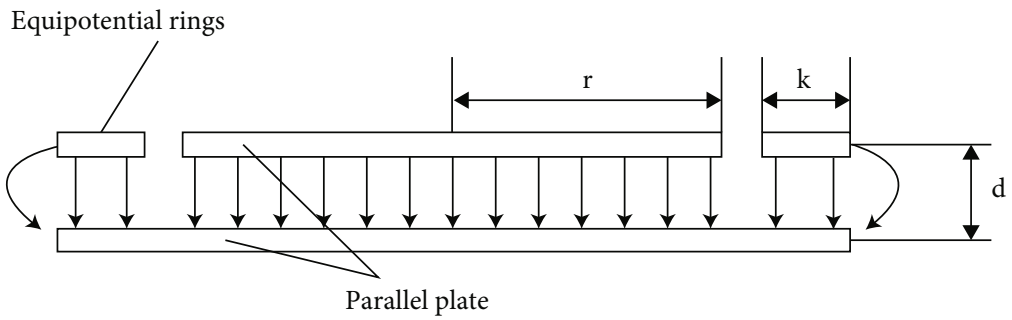

FIgURE 5: Structure diagram of the parallel plate capacitive sensor with the equipotential ring.

perpendicular to the plates, and the lowest signal (zero) will be produced if the electric field is parallel to the plates. So, it is necessary to design the structure of the parallel plate probe and the layout of the probe to form a multidimensional power frequency electric field measurement system.

The shape of our sensing probe is determined based on the tip discharge theory. If a conductor placed under a strong electric field contains a tip or an irregular sharp part, the electric field will be distorted at the tip position and the local electric field will increase. It may even cause partial discharge and affect the measurement of power frequency electric field. Therefore, this paper sets the shape of the parallel plate probe to be circular.

There are other factors that may interfere with the measurement. Because there is an edge capacitance between the parallel plate capacitors, the electric field of the edge effect capacitor is easily interfered by the external electric field. Under the interference of the external electric field, the amount of charge accumulation on the edge of the capacitor is difficult to ensure stability, so the edge effect capacitor is difficult to maintain a constant, thereby forming an edge effect [28]. The edge effect capacitance is part of the total capacitance of the sensor, and its inability to maintain stability will cause uncertainty in the measurement of the sensor, making the output of the sensor unstable, reduced in sensitivity, and nonlinear in the output characteristics. Therefore, in order to reduce the influence of the edge effect when designing the electric field induction unit, the structure of the equipotential ring is adopted to eliminate the edge effect between the two electrode plates.

The design of the unit structure of the parallel plate probe [29] is shown in Figure 5, which consists of three parts: the upper plate, the lower plate, and the equipotential ring. The equipotential ring is in the same plane as the upper pole plate, but there is no electrical connection to the plate. The equipotential ring, the upper pole plate, and the lower pole plate are electrically insulated. This structure can make the field lines at the edge of the upper plate straight, the electric field of the upper and lower plates is basically uniform, and the diverging edge electric field occurs around the periphery of the equipotential ring, so it does not affect the electric field between the two electrodes of the sensing unit, so as to ensure sensor measurement accuracy.

The parallel board probe is made of a printed circuit board (PCB), using a pair of round copper plates as a parallel board, and the insulating material in the middle is epoxy resin. Such a manufacturing process is simple, the cost is low, and the consistency and stability of each unit can be

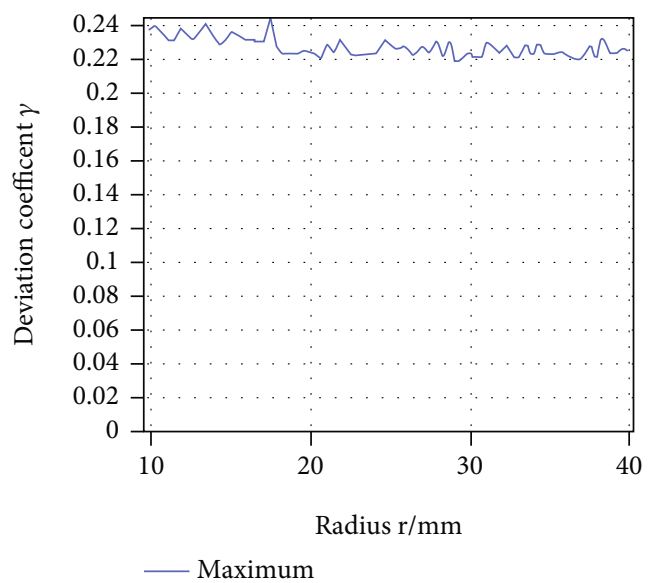

FIGURE 6: The influence of the radius of the induction plate $r$ on the deviation coefficient $\gamma$.

well guaranteed. In addition, the structure is small in size, is easy to integrate, and has good portability.

The electric field simulation tool was used to model and analyze the influence of the structural parameters of the electric field induction unit on the electric field strength. As shown in Figure 5, two parallel plates are built, a power frequency voltage with an effective voltage of $U$ is applied on one parallel plate, the other parallel plate is grounded, and the distance between the two parallel plates is $d$. The electric field intensity generated between the plates is $E=U / d$.

By changing the radius $r$ and spacing $d$ of the induction electrode plate of the electric field induction unit to carry out parametric scanning simulation, the value $E^{\prime}$ of the electric field strength on the induction plate is compared with the original electric field strength $E$.

We use the deviation coefficient

$$
\gamma=\left(E^{\prime}-E\right) / E
$$

to characterize the effect of this structural parameter on the original electric field strength. The simulation results are shown in Figures 6 and 7.

Figure 6 shows the deviation coefficient of the maximum value of the electric field on the induction plates of different radii. The trend of the deviation coefficient numerical curve is generally horiontal, with only a small range of fluctuations. This shows that the change in the area of the induction plate has a small influence on the distortion degree of the induced electric field intensity. But for portability, the sensor 


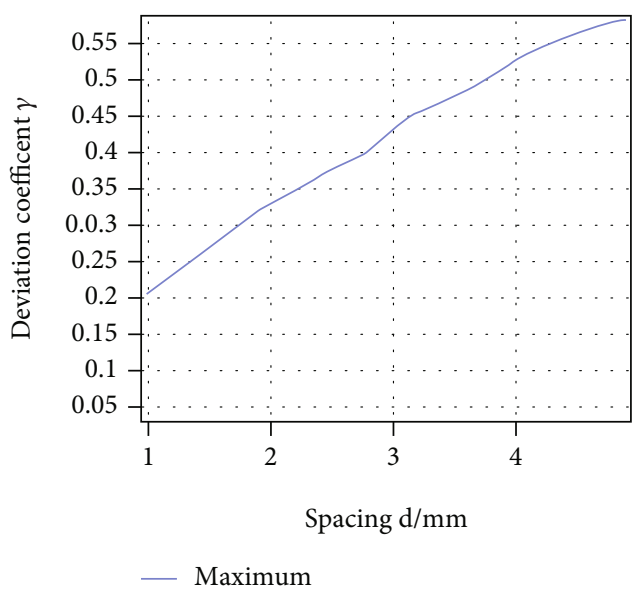

Figure 7: The influence of the distance $d$ between the induction plates on the deviation coefficient $\gamma$.

unit chooses a smaller sheet. In Figure 7, as the distance between the induction plates increases, the deviation coefficient of the maximum and average values of the induced electric field strength also increases linearly. The change in the distance between the induction plates directly affects the degree of distortion of the induced electric field strength. The smaller the thickness, the smaller the distance between the two plates and the closer the electric field intensity induced under the power frequency electric field will be to the electric field intensity at the location of the sensor probe.

As shown in Figure 8, it is an electric field simulation tool simulation diagram for determining the equipotential ring width $k$ of the parallel plate probe.

We use the contour line of the electric field intensity of

$$
E^{\prime}=(1+6 \%) E,
$$

on the induction plate (black line in the figure) and determine the equipotential ring width $k$ of the sensing unit of a different radius according to the width of its contour and the edge of the plate of the sensing unit. As shown in Figure 8 , determining the width of the equipotential ring can exclude $94 \%$ of the distortion electric field.

The parametric scanning simulation is used to calculate the width $k$ of the equipotential ring of the sensor unit with different radii. Several typical inductive plates $r$ are selected. The results for the equipotential ring width $k$ are shown in Table 1 as follows:

It can be seen in Table 1 that when $r$ of the sensing unit continues to increase, the width of the equipotential ring $k$ of the sensing unit first decreases rapidly. After $r$ reaches a certain size, the width of the equipotential ring $k$ tends to stabilize. After the curve fitted by MATLAB, the relationship between $r$ of the sensing unit and the width $k$ of its equipotential ring is

$$
k=8.35 r^{-0.3584} .
$$

3.2. The Actual Test of the Probe Structure of the Measurement System. To reduce the influence of edge effects on the test results and obtain more accurate test results, the parallel plate probe is placed in the calibration device shown in Figure 5. After analyzing the output of the sensing unit through an oscilloscope, the voltage regulator was adjusted to adjust the electric field strength of the uniform electric field and the output voltage value of the AC voltage source was recorded (as shown in Figure 9); after completing the voltage regulation, the output value of the sensing unit was read; after testing one sensing unit, the next one for testing was replaced.

We used the LCR tester to measure the inherent capacitance of the parallel plate probe. The temperature of the LCR test instrument is $24^{\circ} \mathrm{C}$ at the time of measurement, the temperature at the time of measurement is $26^{\circ} \mathrm{C}$, and the temperature drift of the capacitor is $33 \mathrm{ppm} /{ }^{\circ} \mathrm{C}$. The capacity difference caused by this is $C_{\text {diff }}=(24-26) \times 33 \times 10^{-6}=$ $6.6 \times 10^{-5}$; the impact of this $C_{\text {diff }}$ reaching $10^{-5}$ on the output of this system is negligible.

The analysis of the results is shown in Table 2 as follows:

It can be seen in Table 2 that as the sensing area of the sensing unit increases, the sensitivity of the sensing units 1 to 4 continues to increase, while the sensitivity of the sensing units 5 and 6 decreases, in which the sensitivity is close; the reason is still under investigation.

Combining Tables 1 and 2 and the above analysis, the following parameters $r=16 \mathrm{~mm}, k=3.05 \mathrm{~mm}$, and $d=1.6 \mathrm{~mm}$ were chosen for the parallel plate probe. Therefore, the inherent capacitance of the probe is $C_{x}=\varepsilon_{0} \varepsilon_{r} S / d \approx 18.7026 \mathrm{pF}$.

\subsection{Parameter Design of the Differential Integration Circuit.} According to the waveform characteristics of the parallel plate probe output, we only need to extract the power frequency signal to ensure the integrity of the power frequency signal and the high-frequency response and low-frequency response must be less than the power frequency response and the high-frequency characteristic of the measurement system is determined by the differential part and the lowfrequency characteristic is determined by the integral part $[30,31]$. The overall circuit diagram with the added integral part is shown in Figure 10.

where $C_{x}$ and $R$ form the differential unit and $C_{i}$ and $R_{i}$ form the passive first-order integration unit. In this system, when $R$ is small, in the differential circuit part, the voltage across $R$ is

$$
U_{R} \approx R \cdot C_{x} \frac{d U_{\text {in }}(t)}{d t}
$$

Input $U_{R}$ into the integrator, and choose the integral circuit parameters reasonably; you can get the $U_{\text {out }}(t)$ that meets the measurement accuracy requirements and reflects the measured voltage $U_{\text {in }}(t)$.

The relationship between input $U_{\text {in }}$ and output $U_{\text {out }}$ is

$$
U_{\text {out }}(\mathrm{t})=\frac{1}{R_{i} C_{i}} \int_{0}^{t} U_{R}(\tau) d \tau=\frac{R C_{x}}{R_{i} C_{i}} U_{\text {in }}(\mathrm{t})
$$




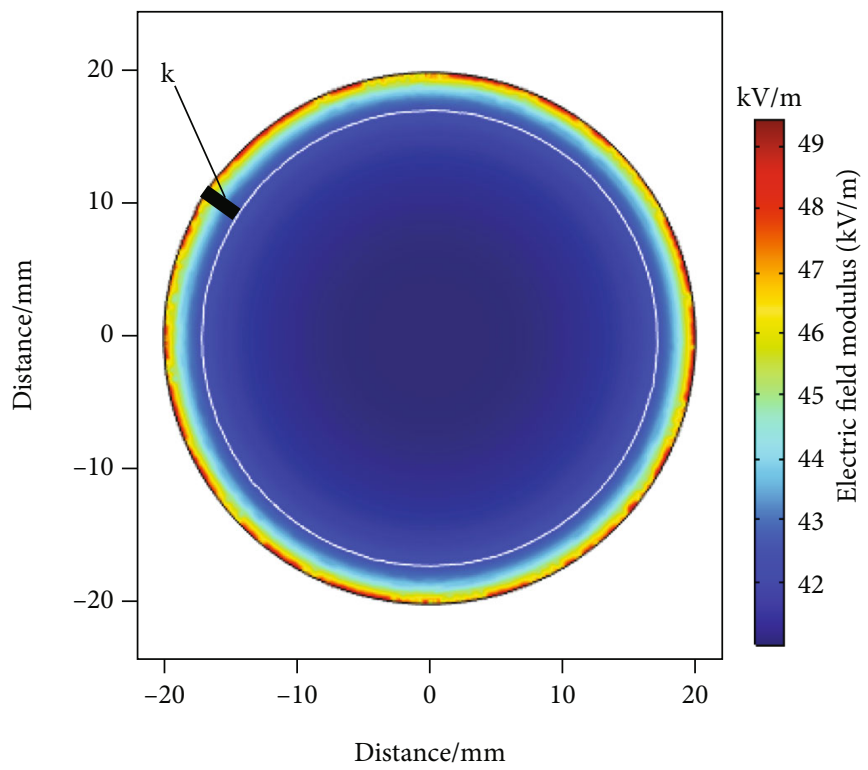

Figure 8: Determination of the width of the equipotential ring of the parallel plate probe.

TABLE 1: Relationship between the radius $r$ of the induction plate and the width $k$ of the equipotential ring.

\begin{tabular}{lcccccc}
\hline Numbering & 1 & 2 & 3 & 4 & 5 & 6 \\
Radius $r$ & 10 & 12 & 14 & 16 & 18 & 20 \\
Width $k$ & 3.8 & 3.4 & 3.2 & 3.05 & 2.95 & 2.9 \\
\hline
\end{tabular}

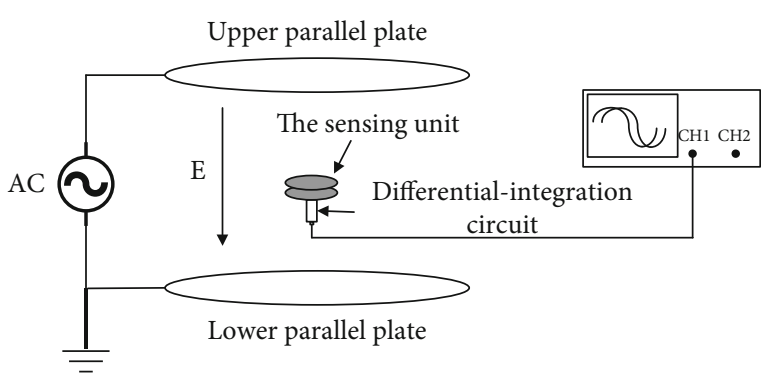

Figure 9: Wiring diagram of measuring the power frequency electric field with a parallel plate probe.

We can get the transfer function of this circuit's voltage:

$$
G(s)=\frac{R}{\mathrm{R}+\left(1 / C_{x} s\right)} \times \frac{1}{R_{i} C_{i} s+1} .
$$

In the actual measurement, due to the complex electromagnetic environment where the power frequency electric field is located, many uncertain factors make the components of the integral and differential parts nonideal. These factors will affect the measurement system, and the parameters of the differentiator and integrator need to be considered in order to eliminate the distortion of the measured electric field waveform caused by higher harmonics and make the power frequency signal output smoothly.

First, design the differential part. The basic structure of the tested system is shown in Figure 9. The front end is a
TABLE 2: Fitting results of measured values of each sensor unit.

\begin{tabular}{lccc}
\hline $\begin{array}{l}\text { Unit } \\
\text { number }\end{array}$ & $\begin{array}{c}\text { Inherent capacitance } \\
\mathrm{Cd}(\mathrm{pF})\end{array}$ & Linearity & $\begin{array}{c}\text { Sensitivity } \\
\left(\mathrm{V} / \mathrm{kV} \cdot \mathrm{m}^{-1}\right)\end{array}$ \\
\hline 1 & 3.01 & $1.81 \%$ & 0.216 \\
2 & 5.79 & $2.88 \%$ & 0.241 \\
3 & 9.12 & $3.09 \%$ & 0.241 \\
4 & 13.02 & $1.60 \%$ & 0.248 \\
5 & 17.84 & $1.61 \%$ & 0.116 \\
6 & 22.88 & $1.64 \%$ & 0.121 \\
\hline
\end{tabular}

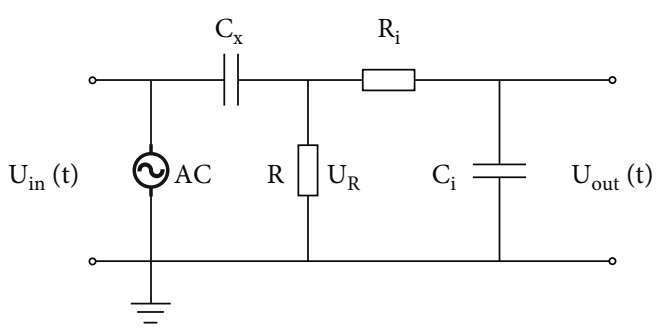

Figure 10: Equivalent calculus circuit diagram.

sensor probe, and the integrator is directly connected to the probe and then passes through the differentiator and connected to the oscilloscope through the coaxial cable. The front-end sensor measures a uniform frequency electric field signal generated by two large flat plates.

In order to study the amplitude-frequency response characteristics of $G(\mathrm{~s})$, according to equation (4), it can be known that a printed circuit board (PCB) is used to make its parallel plate probe, the probe is filled with epoxy resin in the middle, and the material is FR-4, a composite material made by Tera-Function epoxy resin plus filler and glass fiber; its dielectric constant relative to air usually has an range value of 4.2-4.7, the average value $\varepsilon_{r}=4.5$, and $\varepsilon_{0}$ is the vacuum dielectric constant; the value is $8.86 \times 10^{-12} \mathrm{~F} / \mathrm{m}$. Assuming 


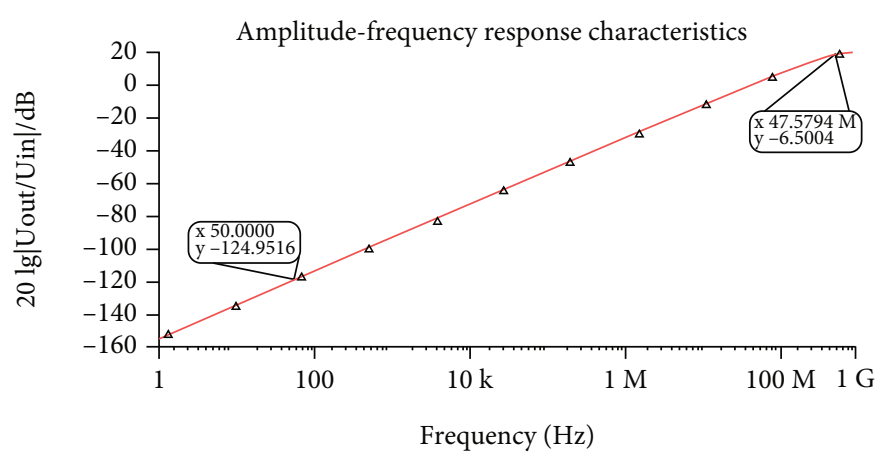

(a)

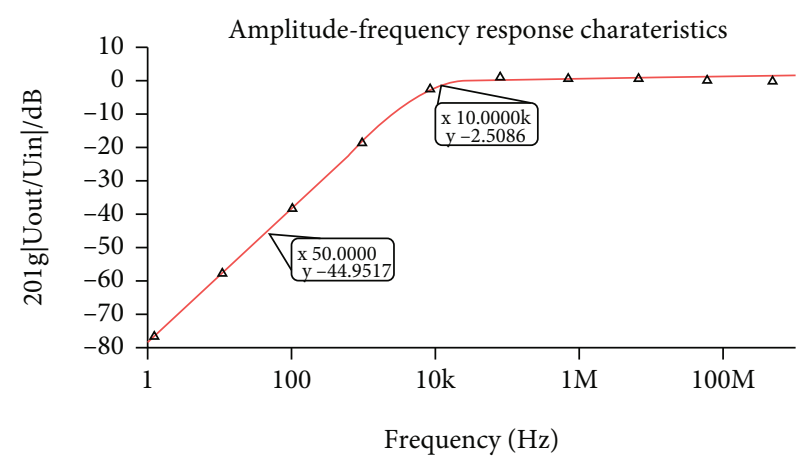

(b)

FIGURE 11: Amplitude-frequency response characteristics of different resistances. (a) Amplitude-frequency response characteristics of the sensor when $R=100 \Omega$ and (b) amplitude-frequency response characteristics of the sensor when $R=1 \mathrm{M} \Omega$.

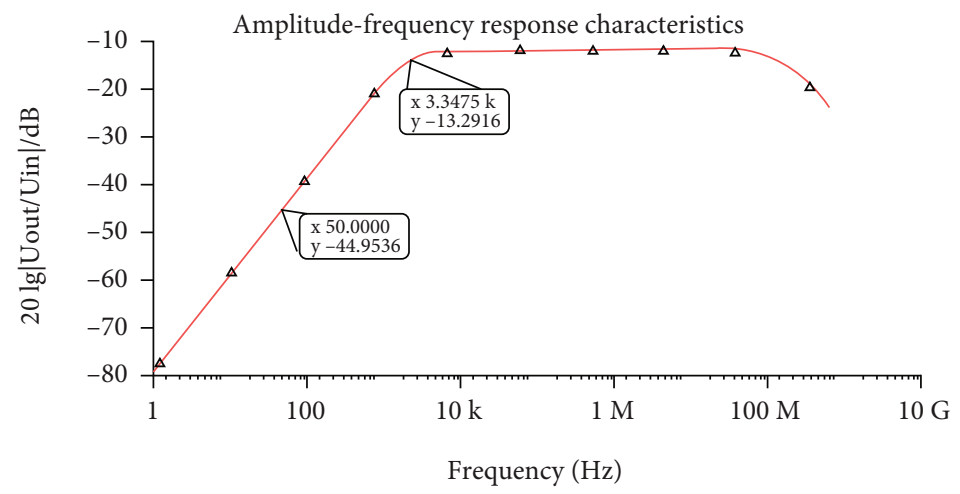

(a)

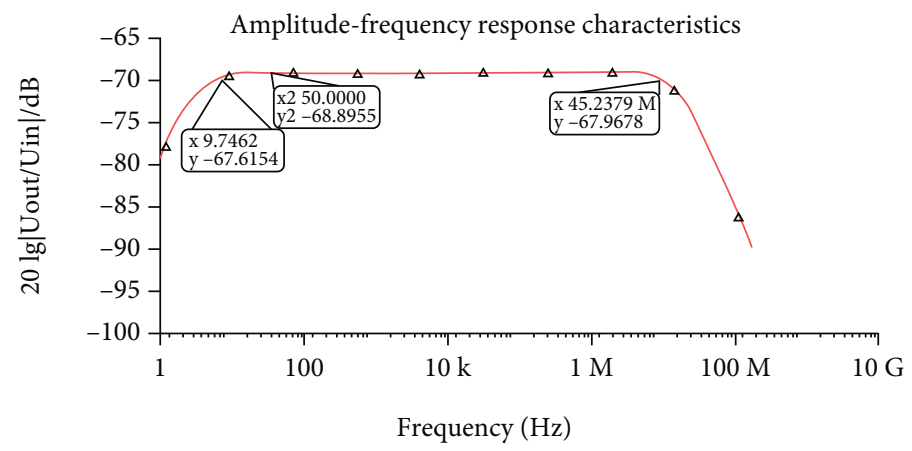

(b)

FIGURE 12: Amplitude-frequency response characteristics under different capacitor sizes. (a) Amplitude-frequency response characteristics of the sensor when $C_{i}=50 \mathrm{pF}$ and (b) amplitude-frequency response characteristics of the sensor when $C_{i}=40 \mathrm{nF}$.

the inherent capacitance of the parallel plate probe $C_{x}=10 \mathrm{pF}$, $R=100 \Omega$, as shown in Figure 11(a).

It can be seen from this that its response at high frequencies is high, which inevitably causes distortion of the output signal, and therefore, the high frequency response needs to be weakened. According to equation (9), it is only necessary to increase the size of $R \times C_{x}$ to achieve this effect. Assuming that the inherent capacitance $C_{x}$ of the parallel plate probe is unchanged, it is only necessary to increase the resistance of $R$ . However, blindly increasing the size of $R$ has little effect on the power frequency response after $R$ is in the megohm level, so set $R=1 \mathrm{M} \Omega$, and its amplitude-frequency response characteristics are shown in Figure 11(b).

For the integration circuit, first assume that $R_{i}=50 \Omega$, $C_{i}=50 \mathrm{pF}$; its amplitude-frequency response characteristics are shown in Figure 12(a).

Similarly, according to Figure $12(\mathrm{a})$, it is necessary to weaken the high-frequency response. According to equation (11), consider increasing $R_{i}$ or increasing $C_{i}$. For this system, we consider increasing $C_{i}$ to weaken the high-frequency response. Therefore, set $C_{i}=40 \mathrm{nF}$. The amplitude-frequency response characteristics are shown in Figure 12(b). 


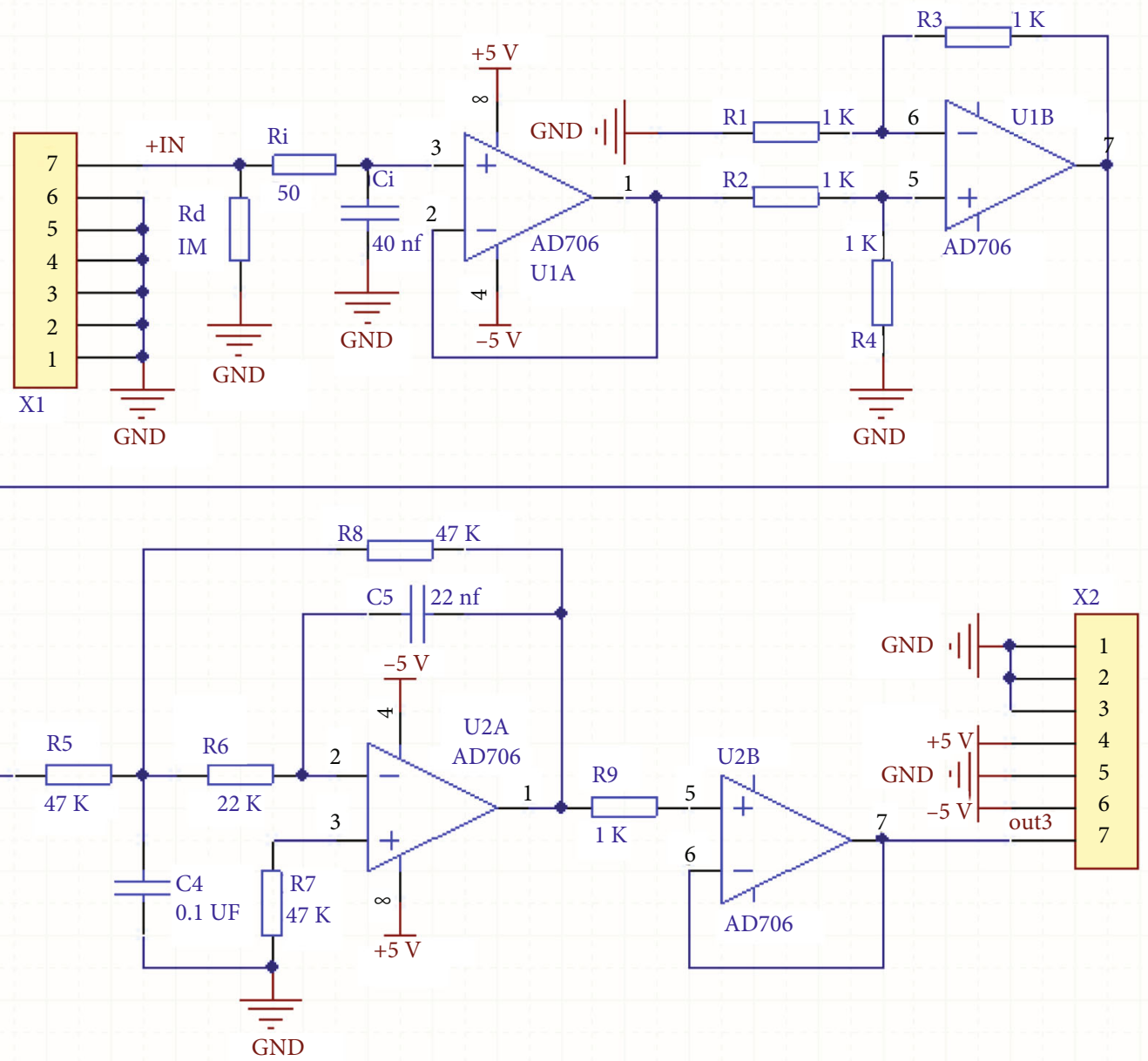

FIGURE 13: Measuring system conditioning circuit principle.

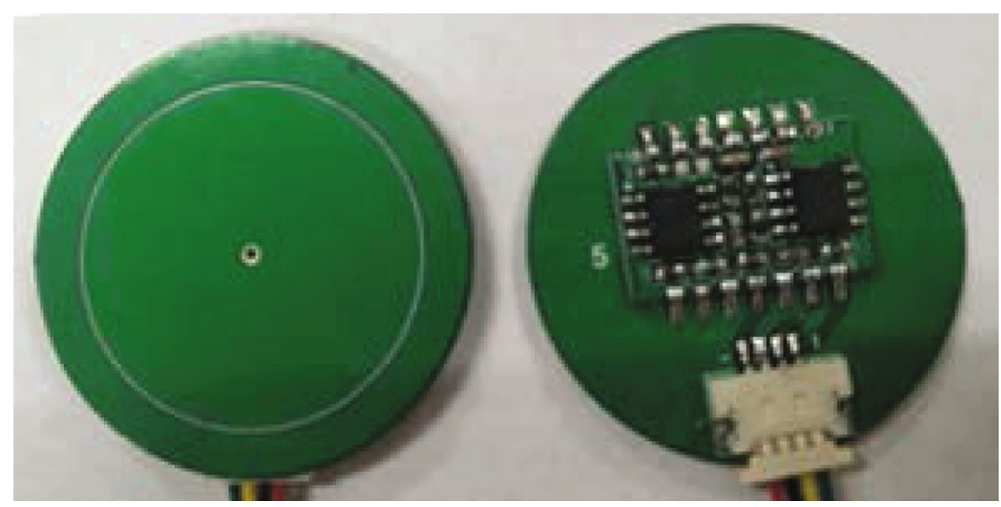

FIgURE 14: Physical diagram of the parallel plate probe and back-end circuit.

3.4. Circuit Design of Measurement System. At the back end of the single-channel parallel plate probe, the signal passes through a differential integration circuit, then through a voltage follower to increase the output impedance, and then use a differential circuit to differentiate the signal. After sending the signal to the full-wave rectifier circuit, we use two capacitors with a large capacitance to output a DC voltage.
The measurement system conditioning circuit principles are shown in Figure 13, and the parallel plate probe and back-end circuit physical diagram are shown in Figure 14.

3.5. Calibration of Flat Capacitive Sensors. For a single-plate capacitive sensor, we need to calibrate it [31] to measure the relationship between input field strength and output voltage under different uniform electric fields. 


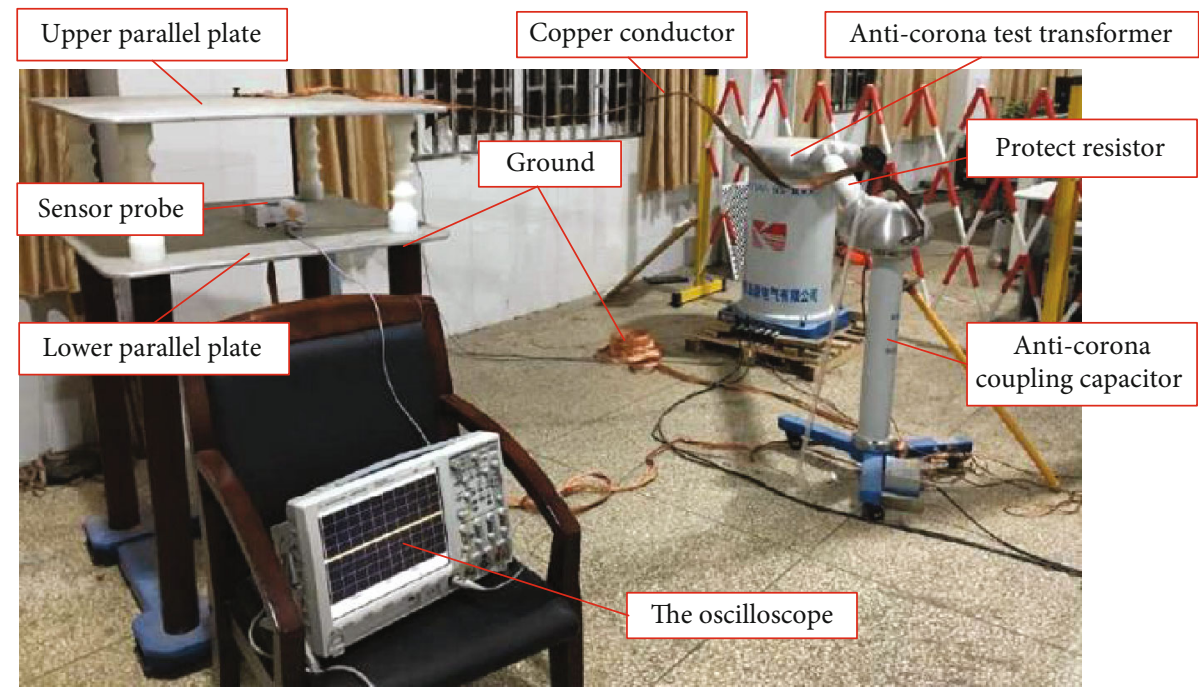

FiguRE 15: $100 \mathrm{kV}$ anticorona test transformer power frequency test platform.

The basic structure used in the calibration system is shown in Figure 9. Two large flat plates produce a uniform electric field signal at power frequency. The power frequency electric field test was conducted on a $100 \mathrm{kV}$ anticorona test transformer. The actual test platform is shown in Figure 15. And the analysis of the experiments result is shown in Figure 16.

The actual test results of the sensor in the high-voltage experimental platform are as shown in Table 3 as follows:

By fitting the curve, the linear relationship between the voltage value $U(t)$ output by each electric field sensor and the uniform electric field $E$ can be obtained. It can also be seen in Table 4 as follows:

The sensitivity coefficients of the sensors designed in this paper are close, the consistency is good, the sensors also have higher linearity, and the linearity fit is up to 0.9998. However, it can also be seen from the experimental data that the sensors made using the PCB process cannot achieve complete consistency. On the one hand, sensors calibrated under the same environment can establish a linear relationship between the output voltage of the sensor and the field strength of the measurement point to obtain the calibration coefficient; on the other hand, the calibration coefficient of each sensor can be used to ensure the accuracy of the sensor when it is measured individually.

\section{Research on the Three-Dimensional Electric Field Measurement System}

4.1. Probe Research of the Three-Dimensional Electric Field Measurement System. Due to the directionality of the electric field, the sensor needs to be able to effectively detect the amount of electric field in different directions. However, a single-plate capacitive sensor can only measure the electric field in one direction. The electric field is a vector, and the electric field value of a single probe cannot accurately reflect the magnitude of the combined electric field. Therefore, capacitive plates were placed on six surfaces of the hexahedron to form six sets of capacitive sensors, so that the electric field strength in different directions could be measured. The probe structure of the electric field measurement system is shown in Figure 17 as follows:.

The multiprobe three-dimensional layout hexahedron method is used to eliminate the electric field measurement uncertainty introduced by the parallel plate without a direct field source. The hexahedron is made of ABS material, which has good electrical insulation and is hardly affected by temperature, humidity, and frequency, and can be used in most environments. The six probes contain conditioning circuits and the smallest single-chip microcomputer system including A/D sampling circuits, which can convert analog signals into digital signals for output. As shown in Figure 18, at a distance of $1.5 \mathrm{~m}$ from the transmission line and parallel to the height of the transmission line, the three-dimensional probe rotates up and down around the $x$-axis. In this paper, we ignore the mutual influence between the probes and assume that the field source is perpendicular to the $x$-axis, where the relationship between sensor 4 probe electric field value $E_{4}$, sensor 5 probe electric field value $E_{5}$, and real electric field $E_{\mathrm{r}}$, as shown in equation (12) as follows:

$$
\begin{gathered}
E_{y}=E_{4}=E_{z} \times \cos \theta, \\
E_{z}=E_{5}=E_{z} \times \sin \theta, \\
E_{r}=\sqrt{E_{y}^{2}+E_{z}^{2}} .
\end{gathered}
$$

Generalize the 2D plane to the three-dimensional space, assuming that the vertical rotation angle is $\theta$ and the horizontal rotation angle is $\delta(\theta$ is the angle between the $y z$ plane and $\delta$ is the angle between the $x y$ plane), the relationship between the combined electric field $E$ and the electric 


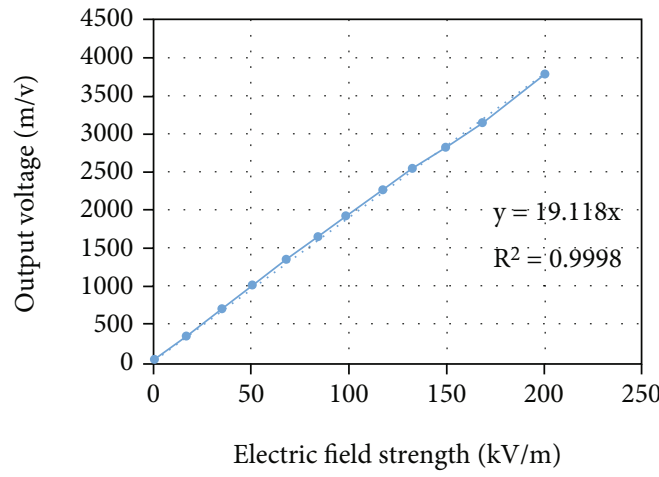

(a)

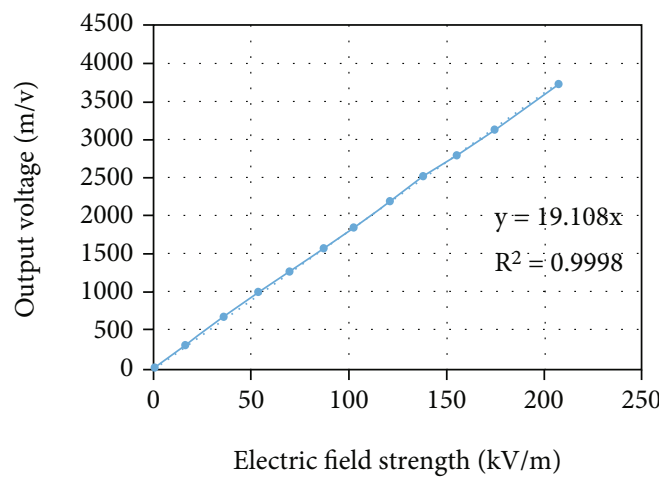

(c)

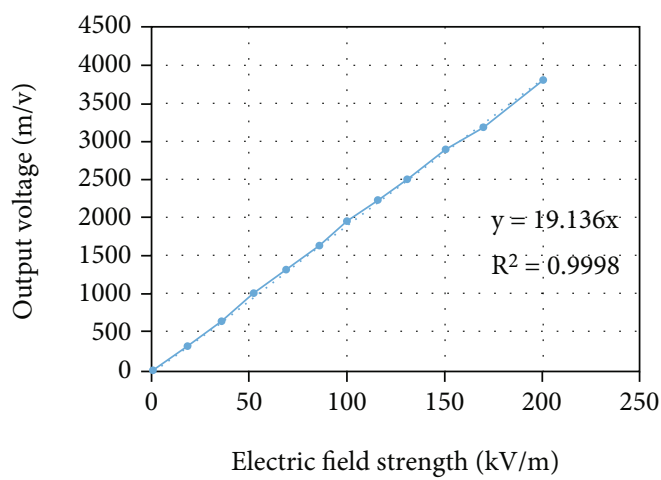

(e)

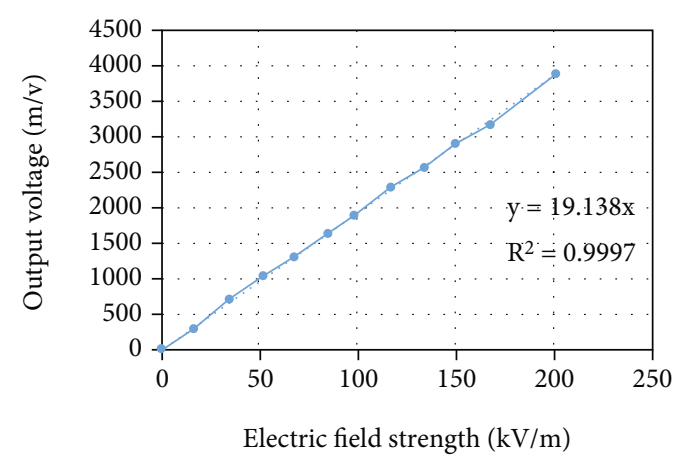

(b)

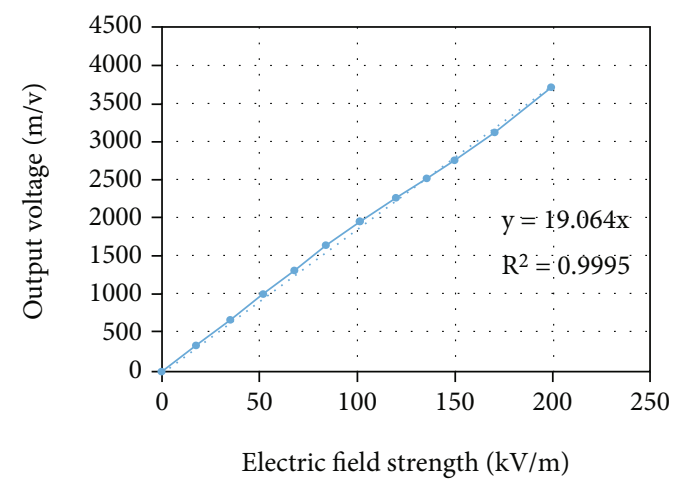

(d)

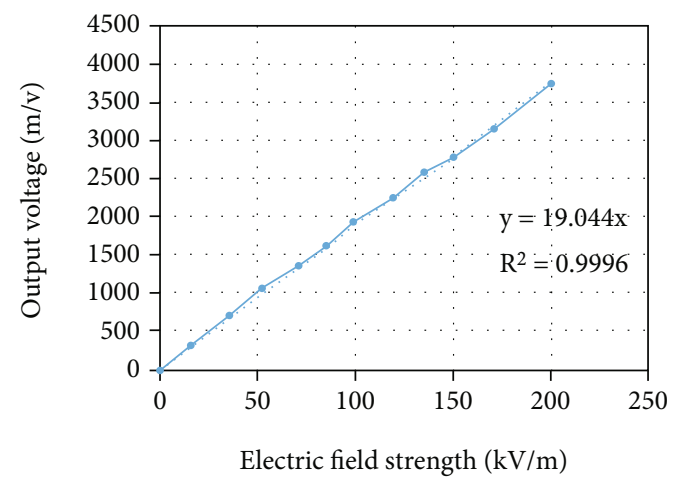

(f)

FIGURE 16: Fitting result of probe calibration data of measurement system. (a) Sensor 1 output, (b) sensor 2 output, (c) sensor 3 output, (d) sensor 4 output, (e) sensor 5 output, and (f) sensor 6 output.

fields $E_{1}, E_{4}$, and $E_{5}$ of probes 1 , probe 4 , and probe 5, respectively, as shown in equation (13) as follows:

$$
\begin{gathered}
E_{X}=E_{1}=E \sin \theta \cos \delta, \\
E_{y}=E_{4}=E \cos \theta \cos \delta, \\
E_{z}=E_{5}=E \sin \delta .
\end{gathered}
$$

And then, the value of combined electric field $E$ can be calculated as follows:

$$
E=\sqrt{E_{x}^{2}+E_{y}^{2}+E_{z}^{2}}
$$

In equation (14), it can be seen that the combined electric field $E$ is not affected by the rotation angles $\theta$ and $\delta$. In the same way, in the eight corners of the cube, each corner has three adjacent sides, so that eight data can be calculated. According to the principle of maximizing, among the eight data, the largest data is the electric field intensity at the location of the sensor. As long as the electric field values in the three directions of $x, y$, and $z$ are obtained, the size of the combined field can be obtained.

4.2. Axial Sensitivity. We use the test platform shown in Figure 15 to test the three-dimensional electric field measurement system. In order to eliminate all other interference, we moved the system to an open place and used lithium 
TABLE 3: Sensor consistency test and calibration data.

\begin{tabular}{|c|c|c|c|c|c|c|c|}
\hline $\begin{array}{l}\text { Input voltage } \\
(\mathrm{kV})\end{array}$ & $\begin{array}{c}\text { Electric field } \\
\text { strength }(\mathrm{kV} / \mathrm{m})\end{array}$ & $\begin{array}{c}\text { Sensor } 1 \\
\text { output }(\mathrm{mV})\end{array}$ & $\begin{array}{c}\text { Sensor } 2 \\
\text { output }(\mathrm{mV})\end{array}$ & $\begin{array}{c}\text { Sensor } 3 \\
\text { output }(\mathrm{mV})\end{array}$ & $\begin{array}{c}\text { Sensor } 4 \\
\text { output }(\mathrm{mV})\end{array}$ & $\begin{array}{c}\text { Sensor } 5 \\
\text { output }(\mathrm{mV})\end{array}$ & $\begin{array}{c}\text { Sensor } 6 \\
\text { output }(\mathrm{mV})\end{array}$ \\
\hline 0.1 & 0.33 & 30 & 21 & 44 & 35 & 38 & 46 \\
\hline 5 & 16.67 & 332 & 308 & 355 & 338 & 340 & 343 \\
\hline 10.5 & 35 & 700 & 723 & 711 & 695 & 688 & 703 \\
\hline 15.4 & 51.33 & 1020 & 1025 & 1013 & 1036 & 1037 & 1041 \\
\hline 20.3 & 67.67 & 1340 & 1311 & 1322 & 1369 & 1323 & 1322 \\
\hline 25.2 & 84 & 1640 & 1634 & 1611 & 1665 & 1618 & 1641 \\
\hline 29.6 & 98.67 & 1920 & 1894 & 1890 & 1929 & 1918 & 1925 \\
\hline 35.1 & 117 & 2260 & 2289 & 2265 & 2251 & 2249 & 2232 \\
\hline 40 & 133.33 & 2550 & 2540 & 2572 & 2543 & 2517 & 2600 \\
\hline 44.9 & 149.67 & 2850 & 2880 & 2860 & 2808 & 2897 & 2829 \\
\hline 50.5 & 168.33 & 3170 & 3143 & 3189 & 3128 & 3175 & 3133 \\
\hline 60.1 & 200.33 & 3802 & 3828 & 3784 & 3810 & 3817 & 3773 \\
\hline
\end{tabular}

Analysis of the above experimental results.

TABLE 4: Linear relationship between the voltage value output by the electric field sensor and the uniform electric field.

\begin{tabular}{lccc}
\hline Sensor & Linear relationship & Sensitivity coefficient, $\mathrm{mV} /\left(\mathrm{kV} \cdot \mathrm{m}^{-1}\right)$ & Degree of fit \\
\hline Sensor 1 & $y=19.118 x$ & 19.118 & 0.9998 \\
Sensor 2 & $y=19.138 x$ & 19.138 & 0.9997 \\
Sensor 3 & $y=19.108 x$ & 19.108 & 0.9998 \\
Sensor 4 & $y=19.064 x$ & 19.064 & 0.9995 \\
Sensor 5 & $y=19.136 x$ & 19.136 & 0.9998 \\
Sensor 6 & $y=19.044 x$ & 19.044 & 0.9996 \\
Average value & - & 19.101 & 0.9997 \\
Mean absolute deviation & - & 0.031556 & 0.0001 \\
\hline
\end{tabular}

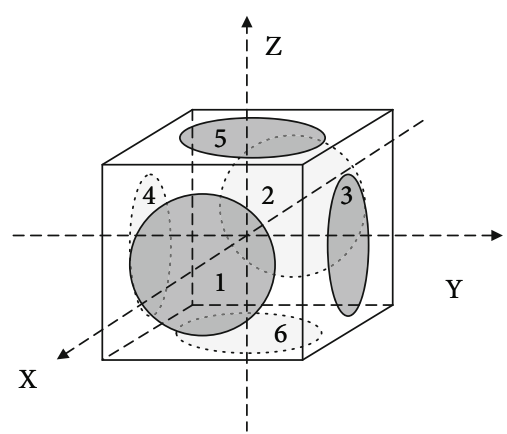

(a)

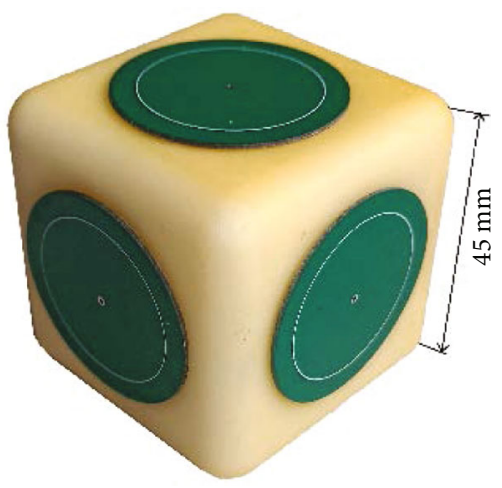

(b)

Figure 17: (a) Probe structure diagram of the electric field measurement system. (b) Physical image of the three-dimensional electric field sensor.

batteries for the power supply. When testing the system, the data shown in the following table is obtained.

After testing, according to Table 5, we can clearly find that the data measured in the $y$-axis and $z$-axis directions is obviously close to 0 when the $x$-axis face 1 is facing upward. The combined field strength obtained in its three directions conforms to the preset field strength. The same results are obtained for the $y$-axis and the $z$-axis.

\section{Field Measurement}

In working conditions, the electric field environment is complex and changeable and the distortion of the electric field in the sensor cannot be measured. Therefore, the mathematical relationship obtained through theoretical analysis may deviate in working conditions. In order to ensure the normal operation of the electric field sensor in the environment with 


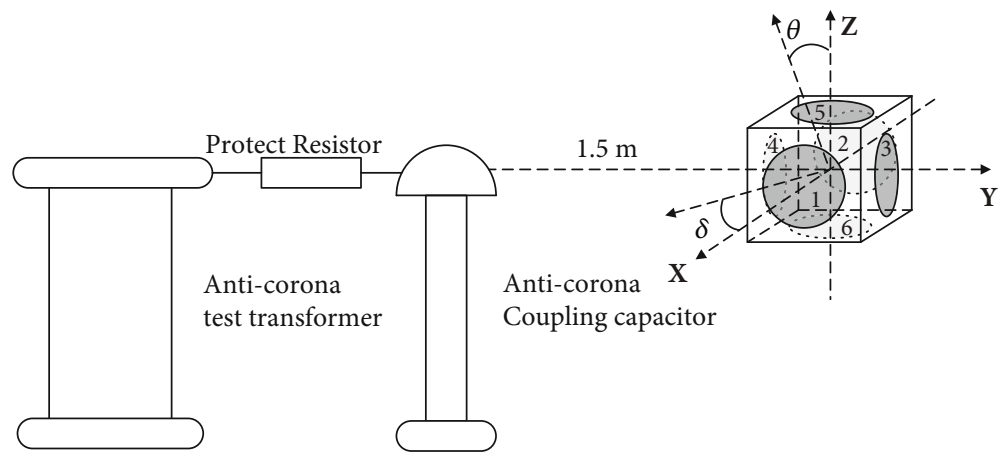

FIGURE 18: Schematic diagram of the deflection angle.

TABLE 5: Axial sensitivity test.

\begin{tabular}{lcccc}
\hline Field strength is $1000 \mathrm{~V} / \mathrm{m}$ & Face $1: x$-axis $(\mathrm{V} / \mathrm{m})$ & Face $2: y$-axis $(\mathrm{V} / \mathrm{m})$ & Face $3: z$-axis $(\mathrm{V} / \mathrm{m})$ & Field strength $(\mathrm{V} / \mathrm{m})$ \\
\hline Face 1 facing up & 999.5 & 0.5 & 0.2 & 999.5 \\
Face 2 facing up & 0.4 & 1000.3 & 0.1 & 1000.3 \\
Face 3 facing up & 0.5 & 0.3 & 1000.3 & 1000.3 \\
\hline
\end{tabular}

TABle 6: Performance test form.

\begin{tabular}{lc}
\hline Measuring field strength & Range \\
\hline Measuring range & $1 \mathrm{kV} / \mathrm{m}-200 \mathrm{kV} / \mathrm{m}$ \\
Minimum resolution & $\geq 1 \mathrm{~V} / \mathrm{m}$ \\
Overload limit & $200 \mathrm{kV} / \mathrm{m}$ \\
\hline
\end{tabular}

large electric field stress, the designed electric field sensor was tested for stability, sensitivity, and linearity. Therefore, a comparative test experiment is carried out in the same environment using the electric field measurement equipment with higher accuracy and better sensitivity. The NBM-550 broadband field strength meter and the supporting EHP-50F of Narda Company in Germany are the electromagnetic field measuring instruments with higher recognition in the industry. The performance of the electric field sensor such as measurement range, resolution, sensitivity, and linearity is tested by using the calibration platform NBM-550. The performance test table is shown in Table 6.

During the experiment, the EHP-50F and the threedimensional power frequency electric field measurement system were simultaneously separated from the field source by $2.5 \mathrm{~m}$. As shown in Figure 18, the nonpartial discharge transformer is set to $35 \mathrm{kV}$ and the EHP-50F approaches the field source at $0.2 \mathrm{~m}$ steps at a time and records the distance and the corresponding electric field value. Then, let the three-dimensional power frequency electric field measurement system step in the same way to approach the field source at the same position every $0.2 \mathrm{~m}$ and record the corresponding data at the same time. We use the electric field simulation tool to simulate the scene shown in Figure 18 and obtain the field strength value of the corresponding position. The result is shown in Table 7 .

Compare the measurement results of both Narda electric field measuring instrument and the designed electric field sensor and according to the following equation:
TABLE 7: Comparison between EHP-50F and 3D power frequency electric field measurement system as function of the distance.

\begin{tabular}{lcccc}
\hline $\begin{array}{l}\text { Distance } \\
(\mathrm{m})\end{array}$ & $\begin{array}{c}\text { Simulation } \\
(\mathrm{V} / \mathrm{m})\end{array}$ & $\begin{array}{c}\text { EHP_F } \\
50 \mathrm{~V} / \\
\mathrm{m})\end{array}$ & $\begin{array}{c}\text { Three-dimensional } \\
\text { electric field } \\
\text { measurement system } \\
(\mathrm{V} / \mathrm{m})\end{array}$ & $\begin{array}{c}\text { Deviation } \\
(\%)\end{array}$ \\
\hline 0.1 & 24708.55 & 24709 & 24689 & $-0.08 \%$ \\
0.3 & 8235.66 & 8236 & 8271 & $0.43 \%$ \\
0.5 & 4941.28 & 4942 & 4954 & $0.26 \%$ \\
0.7 & 3529.37 & 3530 & 3513 & $-0.46 \%$ \\
0.9 & 2745.66 & 2745 & 2750 & $0.16 \%$ \\
1.1 & 2246.5 & 2246 & 2239 & $-0.33 \%$ \\
1.3 & 1899.56 & 1901 & 1896 & $-0.19 \%$ \\
1.5 & 1647.94 & 1647 & 1639 & $-0.54 \%$ \\
1.7 & 1453.57 & 1454 & 1453 & $-0.04 \%$ \\
1.9 & 1300.62 & 1301 & 1295 & $-0.43 \%$ \\
2.1 & 1176.39 & 1177 & 1180 & $0.31 \%$ \\
2.3 & 1073.86 & 1074 & 1069 & $-0.45 \%$ \\
2.5 & 988.55 & 989 & 988 & $-0.06 \%$ \\
\hline
\end{tabular}

Deviation $=\frac{(\text { sensor measurement })-(\text { simulation })}{(\text { simulation })}$.

After analysis, compared with Narda's EHP-50F and simulation, the highest uncertainty of the threedimensional electric field measurement system developed in this paper is within $\pm 0.55 \%$. As shown in Table 7 , the measurement range is $1 \mathrm{kV} / \mathrm{m}-200 \mathrm{kV} / \mathrm{m}$, the resolution is $\geq 1 \mathrm{~V} / \mathrm{m}$, and the maximum electric field can be measured at $200 \mathrm{kV} / \mathrm{m}$. The nonlinear error is $2.15 \%$, and the sensitivity coefficient is $19.10 \mathrm{mV} /\left(\mathrm{kV} \cdot \mathrm{m}^{-1}\right)$. Limited by the size of $C_{i}$, the greater the $C_{i}$, the greater the limit of the entire output signal, which can reach up to $200 \mathrm{kV} / \mathrm{m}$. Meet the requirements of engineering applications. 


\section{Conclusions}

We designed and optimized a three-dimensional power frequency electric field measurement system, which consists of a sensor probe, a differential integration circuit, an adjustment circuit, and a back-end processing system. The system satisfies the measurement of a three-dimensional power frequency electric field and has a simple structure, convenient manufacture, and high precision. The research results of this article are summarized as follows:

(1) When the parallel plate is used as the probe of the industrial frequency electric field sensor, a differential integration circuit needs to be added to the output of the parallel plate to ensure that the waveform output by the probe is not distorted

(2) In the process of probe design, the use of the equipotential ring can reduce the influence of the edge electric field on the measurement; the relationship between the radius $r$ of the sensing unit and the width $k$ of the equipotential ring satisfies $k=8.35$ $R^{-0.3584}$

(3) When designing the sensor in three dimensions, it is proposed to use the vector sum of field strength equation $E=\sqrt{E_{x}+E_{y}+E_{z}}$ and design the sensor probe with a hexahedral structure. This solves the problem of the inaccurate measurement electric field caused by the parallel plate probe and the field source not being perpendicular

(4) The system can be applied to some newer platforms, such as power helmets, drones, and substation ground inspection robots, to provide them with electric field information accurately, thereby ensuring the safety of live working robots and power workers

(5) It is tested in a laboratory environment and compared with simulation. The uncertainty of the three-dimensional power frequency electric field measurement system is within $\pm 0.55 \%$, the measurement range is $1 \mathrm{kV} / \mathrm{m}-200 \mathrm{kV} / \mathrm{m}$, the resolution is $\geq 1 \mathrm{~V} / \mathrm{m}$, and the maximum electric field can be measured at $200 \mathrm{kV} / \mathrm{m}$. The nonlinear error is $2.15 \%$, and the sensitivity coefficient is $19.10 \mathrm{mV} /\left(\mathrm{kV} \cdot \mathrm{m}^{-1}\right)$, which meets the power frequency electric field measurement requirements and can be applied to the actual power frequency electric field measurement

\section{Data Availability}

The data used to support the findings of this study are included within the article.

\section{Conflicts of Interest}

The authors declare that there is no conflict of interest regarding the publication of this paper.

\section{Acknowledgments}

This research was supported by the "Research and development of new smart sensor technology to promote the development of green energy" (202104BN050011) funding.

\section{References}

[1] B. Sun, Research on Integrated Optical Electromagnetic Field Sensor, [Ph. D. Thesis], University of Electronic Science and Technology, Chengdu, 2010.

[2] W. Zhang, P. Li, N. Zhou et al., "Method for localization aerial target in AC electric field based on sensor circular array," Sensors, vol. 20, no. 6, 2020.

[3] W. Zhu, J. Guo, G. Zhang, D. Yao, S. Zhang, and S. Qian, "Research and design of power frequency electric field measurement system," Procedia Computer Science, vol. 155, pp. 740-745, 2019.

[4] C. Suo, H. Sun, W. Zhang, N. Zhou, and W. Chen, "Adaptive safety early warning device for non-contact measurement of HVDC electric field," Electronics, vol. 9, no. 2, 2020.

[5] H. Wang, R. Zeng, C. Zhuang et al., "Measuring AC/DC hybrid electric field using an integrated optical electric field sensor," Electric Power Systems Research, vol. 179, 2020.

[6] T. Zhang, Z. Fang, T. Chen, and L. Zhao, "Application of spherical electric field measurement system in the field of high voltage measurement," Electrical Measurement and Instrumentation, vol. 44, no. 11, pp. 11-16, 2007.

[7] P. Hu, Research and Application of Spherical Electric Field Sensor Measurement System, Chongqing University, Chongqing, 2011.

[8] P. Nie, Y. Cui, H. Yuan, G. Wu, and Y. Zhao, "A power frequency electric field sensor based on the principle of differential conduction current," Measurement and Control Technology, vol. 1, pp. 1-3, 2015.

[9] C. H. Bulmer, W. K. Burns, and S. C. Hiser, "Pyroelectric effects in LiNbO3 channel-waveguide devices," Applied Physics Letters, vol. 1, no. 48, pp. 1036-1038, 1986.

[10] R. C. Alferness, "Waveguide electropitic modulators," IEEE Trans on Microwave Theory and Techniques, vol. 30, no. 8, pp. 1121-1137, 1982.

[11] K. Hidaka, T. Kouno, and I. Hayashi, "Simultaneous measurement of two orthogonal components of electric field using a Pockels device," The Review of Scientific Instruments, vol. 60, no. 7, pp. 1252-1257, 1989.

[12] D. Ren, C. Peng, and S. Xia, "Novel wireless electrical system based on LabVIEW and MEMS electric-field sensor to measure power-frequency electric field," Instrument Technology and Sensor, vol. 1, pp. 53-56, 2014.

[13] D. Ren, C. Peng, and S. Xia, "Micromachined electric-field sensor system based on direct digital synthesizer and lock-inamplifier to measure AC and DC fields," Journal of Electronics and Information Technology, vol. 12, pp. 3030-3036, 2013.

[14] P. Yang, B. Chen, X. Wen, C. Peng, S. Xia, and Y. Hao, "A novel MEMS chip-based atmospheric electric field sensor for lightning hazard warning applications," in 2015 IEEE SENSORS, Busan, 2015.

[15] K. Feser and W. Pfaff, "A potential free spherical sensor for the measurement of transient electric field," IEEE Trans. PAS, vol. 103, no. 10, pp. 2904-2911, 1984. 
[16] E. Gockenbach, "A new measuring device for transient over voltages," CIGRE Symposium New and Improved Materials for Electrotechnology., pp. 1010-1015, 1987.

[17] J. Ramirez, "A device for the X- Y measurement of electric fields," Measurement Science Technique, vol. 5, no. 5, pp. 1436-1442, 1994.

[18] W. R. PFaff, "Accuracy of a spherical sensor for the measurement of three-dimensional electric fields," Fifth International Symposium on High Voltage Engineering., vol. 32, no. 5, 1987.

[19] X. Wen, C. Peng, D. Fang et al., "High performance electric field micro sensor with combined differential structure," Journal of Electronics, vol. 31, no. 2, pp. 143-150, 2014.

[20] R. Qin, Z. Yang, L. An, J. Lei, and Y. Yang, "Design of high voltage electric field measurement based on parallel plate sensor," Electrical Application, vol. 19, pp. 72-78, 2016.

[21] L. Chen, W. Wang, F. Lu, X. Yuan, and H. Shen, "Portable wireless power frequency electric field sensor network," Instrument Technology and Sensor, vol. 5, no. 5, pp. 5-15, 2018.

[22] F. Zhao, Q. Zhang, X. Cai, L. Fan, and Y. Sun, “The questions in measure of power frequency electromagnetic field," The Administration and Technique of Environmental Monitoring, vol. 5, pp. 57-58, 2007.

[23] C. Yang, Research on Voltage Level Automatic Identification Method of High Voltage Transmission Line Based on SVM, Kunming University of Science and Technology, 2018.

[24] G. Jiang and J. Yang, "Power frequency electric field measurement and the study of a new type measuring probe," High Voltage Engineering, vol. 4, pp. 1-5, 1985.

[25] J. E. Toney, A. Pollick, J. Retz, and S. Sriram, "Noncontact electro-optic near field probe for surface electric field profiling," in 2016 IEEE SENSORS, Orlando, FL, 2016.

[26] D. Kumar, N. R. Prakash, and S. Singh, "Electric field sensor for electromagnetic pulse measurement," IETE Technical Review, vol. 36, no. 6, 2019.

[27] K. M. Bohnert, H. Brändle, and G. Frosio, "Field test of interferometric optical fiber high-voltage and current sensors," Tenth International Conference on Optical Fibre Sensors, vol. 2360, 1994.

[28] K. Xiong and D. Ni, "Edge effect of capacitive sensor," Sensor World, vol. 3, pp. 16-19, 1998.

[29] L. Fan and W. Zhang, "Design and analysis of power frequency electric field sensing unit with reduce edge effect," Journal of Physics: Conference Series, vol. 1213, no. 4, 2019.

[30] W. Zhang, "The influence of loop parameters of calculus system on the accuracy of measuring impulse voltage," Journal of Xi'an Jiaotong University, vol. 3, pp. 24-29, 1995.

[31] L. Wang, W. Zhang, X. Tan, W. Chen, S. Liang, and C. Suo, "Research and experiments on an external miniaturized VFTO measurement system,” Sensors, vol. 20, no. 1, 2019. 